\section{Reduction of Redundant Data in the Quadratic Cost Formulation for Linear Time-Variant Systems}

\section{E. BRUCE LEE AND WU-SHENG LU}

Abstract-Time-variant systems represented by pairs of matrices $(A(t), B(t))$ and $(\bar{A}(t), \bar{B}(t))$ are said to be $F$-equivalent if there exist differentiable matrices $C, G$, and $D$ such that $\bar{A}=C^{-1}[(A+B G)-$ $\left.\dot{C} C^{-1}\right] C, \bar{B}=C^{-1} B D$ and $K$-equivalent if $G \equiv 0$. The extent of independent parameters (functions) in the quadratic cost formulation for a linear time-variant system is reported. For the $K$-equivalent class and the $F$ equivalent class upper bounds on the number of independent parameters are explicitly given. The single input quadratic cost formulation contains exactly $n$ independent parameters (functions).

\section{INTRODUCTION}

Several previous studies of redundant data in the quadratic cost formulation for linear systems have been conducted [1]-[8]. Essentially, one seeks a canonical form for the quadratic cost formulation so that important features of questions of optimization are more transparent and there can be groupings into equivalence classes.

The quadratic cost formulation will be considered from two points of view here. In the first case the controlled input will be a prescribed function and we ask the extent of independent parameters in the quadratic cost formulation (reduction to essential parameters in the $K$-equivalence class). In the second case the controlled input is to minimize the quadratic cost, which means that we seek a description of the extent of independent parameters in the $F$-equivalence class (reduction under the optimal feedback group).

Consider a linear time-variant system with mathematical model

$$
\dot{x}(t)=A(t) x(t)+B(t) u(t), \quad t_{0} \leqslant t \leqslant t_{1}, \quad x\left(t_{0}\right)=x_{0}
$$

and with cost functional

$$
J=\int_{t_{0}}^{t_{1}}\left[x^{\prime} Q(t) x+2 x^{\prime} S(t) u+u^{\prime} R(t) u\right] d t+x^{\prime}\left(t_{1}\right) F x\left(t_{1}\right)
$$

where $A(t), B(t), Q(t)=Q^{\prime}(t), S(t)$ and $R(t)=R^{\prime}(t)>0$ are sufficiently differentiable for $t_{0} \leqslant t \leqslant t_{1} . x(t)$ is an $n$-vector and $u(t)$ is an $m$-vector. $F=F^{\prime}$.

When $u(t)$ is a prescribed function in $L_{2}\left(\left[t_{0}, t_{1}\right] ; R^{m}\right)$, we ask for the extent of independent parameters in the calculation of $J(u)=$ $J\left(u, t_{0}, t_{1}, x_{0}, A, B, Q, R, S, F\right)$ and when $u^{*}(t)$ in $L_{2}\left(\left[t_{0}, t_{1}\right] ; R^{m}\right)$ minimizes $J$ we ask for the extent of independent parameters of $J\left(u^{*}\right)$.

As in [9], two time-variant systems as represented by their coefficient matrices $(A(t), B(t))$ and $(\bar{A}(t), \bar{B}(t))$ are said to be: 1$) F$-equivalent, if there are differentiable matrices $C(t), G(t), D(t)$ on $\left[t_{0}, t_{1}\right]$, such that $\bar{A}(t)=C^{-1}\left[(A+B G)-\dot{C} C^{-1}\right] C$ and $\bar{B}(t)=C^{-1} B D$, for $\left.t \in\left[t_{0}, t_{1}\right] ; 2\right)$ $C$-equivalent, if $G \equiv 0$ and $\left.D=I_{m} ; 3\right) G$-equivalent, if $C=I_{n}$ and $D=I_{m}$; 4) $D$-equivalent, if $C=I_{n}$ and $G \equiv 0$; and 5) $K$-equivalent, if $G \equiv 0$.

$F$-equivalence is an equivalence relation and so are the $C-, G-, D$-, $K$-equivalences.

Because of the $R(t)>0$ restriction in the $D$-equivalence class, the transformation $R^{1 / 2} u \rightarrow u$ will lead to a cost functional with $R=I_{m}$ :

$$
\int_{t_{0}}^{t_{1}}\left[x^{\prime} Q(t) x+2 x^{\prime} S(t) u+u^{\prime} u\right] d t+x^{\prime}\left(t_{1}\right) F x\left(t_{1}\right)
$$

a first reduction of redundant data. In $K$-equivalence class there exists the possibility of further reducing redundant data among $\{A, B, Q, S, F\}$ in

\footnotetext{
Manuscript received March 21, 1983; revised August 5, 1983. This work was supported by the National Science Foundation under Grant ECS 82-17375.

The authors are with the Department of Electrical Engineering, University of Minnesota, Minneapolis, MN 55455.
}

the calculation of $J(u)$ and in the $F$-equivalence class there exists the possibility of further reducing redundant data of $J\left(u^{*}\right)$.

The minimal optimal solution can be obtained by using the state feedback controller ${ }^{1} u^{*}(t)=-K(t) x(t)$ with $K(t)=B^{\prime}(t) P(t)+S^{\prime}(t)$ where $P(t)$ is the symmetric positive definite solution of Riccati differential equation $-\dot{P}(t)=A^{\prime} P+P A+Q-(P B+S)(P B+S)^{\prime}$ with $P\left(t_{1}\right)$ $=F$.

Let $\bar{P}(t)=P(t)+L(t)$ where $L(t)$ is any known, differentiable, and symmetric $n \times n$ matrix, then the Riccati equation is $-\bar{P}(t)=A^{\prime} \bar{P}+\bar{P} A$ $+\bar{Q}-(\bar{P} B+\bar{S})(\bar{P} B+\bar{S})^{\prime}$, with $\bar{P}\left(t_{1}\right)=F+L\left(t_{1}\right)$ where $\bar{Q}=Q-A^{\prime} L$ $-L A-\dot{L}$, and $\bar{S}=S-L B$.

The feedback gain matrix $K(t)$ does not change when one replaces $P$ and $S$ by $\bar{P}$ and $\bar{S}$, respectively. The quadratic optimization formulation thus has its equivalent description with the cost functional

$\int_{t_{0}}^{t_{1}}\left[x^{\prime} \bar{Q}(t) x+2 x^{\prime} \bar{S}(t) u+u^{\prime} u\right] d t+x^{\prime}\left(t_{1}\right) \bar{F} x\left(t_{1}\right), \quad \bar{F}=F+L\left(t_{1}\right)$.

In the next section, with restriction to the $K$-equivalence class, upper bounds on the number of the independent parameters for the cases $S(t) \equiv 0$ and $S(t) \neq 0$ are given. It is apparent that the Brunovsky canonical form [9] for the linear time-yariant system is an appropriate starting point for our purpose. The technique suggested in [7] is then used to eliminate $(n-m)(n-m+1) / 2$ functions from $Q(t)$ (when $S(t) \equiv 0$ ) or even more if $S(t) \neq 0$. Afterwards, one could eliminate more functions from $A(t)$ which was already in its Brunovsky canonical form by using a simple nonsingular transformation whenever $\operatorname{rank}(B, A B)<2 m$ where $A B \equiv A(t) B(t)+\dot{B}(t), m=\operatorname{rank} B=$ dimension of the input space. This result answers a conjecture raised in [7] and gives some insight into the relationship between Kronecker indexes and independent parameters in the quadratic optimization formulation. A similar situation for the $F$ equivalent class is discussed in Section III. The technique used in [7] and the availability of state feedback and the Riccati equation transformation will considerably cancel dependent functions in $A(t), Q(t)$, and $S(t)$.

\section{Independent Parameters in the $K$-Equivalent Class}

From now on the system is assumed to be controllable, i.e., $\operatorname{rank}\left[B, \dot{A} B, \cdots, \dot{A}^{n-1} B\right]=n$ for $t \in\left[t_{0}, t_{1}\right]$. Also, we assume that $B(t)$ is of full rank for $t \in\left[t_{0}, t_{1}\right]$. We define $R\langle A, B\rangle$ as the $n$-tuple of functions $\left\{r_{j}(t), j=0,1, \cdots, n-1\right\}$ on $\left[t_{0}, t_{1}\right]$ where $r_{j}(t)=\operatorname{rank}\left[B, \cdots, \AA^{j} B\right]-$ $\operatorname{rank}\left[B, \cdots, \mathscr{A}^{j^{-1}} B\right]$. With these notations, it is seen that $B(t)$ being of full rank means $r_{0}=m$.

Suppose a pyramidal basis $S$ defined in [9] has been chosen, one then could associate with every column $b_{i}$ of $B(t)$ a number $p_{i}$ for each $t \in\left[t_{0}, t_{1}\right]$ such that $\AA^{j} b_{i} \in S$ for $0 \leqslant j \leqslant p_{i}-1$ but $\AA^{p_{i}} b_{i} \notin S$. By [9, Lemma 7] it is possible to reorder suitably the columns of $B$ ( $D$-equivalent) so that $p_{1} \geqslant p_{2} \geqslant \cdots \geqslant p_{m}$. Consequently, the $p$-functions could be uniquely determined by the $r$-functions as follows: $p_{i}(t)$ is the function which, at time $t$, is the number of $r_{j}$ 's which are greater than or equal to $i$.

Lemma 2.I: If $r_{1}<r_{0}$, the matrix $B(t)$ then has the form

$$
B(t)=\left[\begin{array}{c}
B_{1}(t) \\
B_{2}(t) \\
\vdots \\
B_{m}(t)
\end{array}\right]=\left[\begin{array}{c}
B_{1} \\
B_{2} \\
\vdots \\
B_{r_{1}} \\
\ldots \ldots \\
0_{m-r_{1} ; r_{1}}: I_{m-r_{1}}
\end{array}\right]
$$

where for each $i \leqslant j \leqslant m, B_{j}$ is defined as a $p_{j} \times m$ matrix with $\left(B_{j}\right)_{j k}=0$ if $j<p_{j},\left(B_{j}\right)_{p_{j} k}=0$ if $k<j .\left(B_{j}\right)_{p_{j} j}=1,\left(B_{j}\right)_{p_{j} k}$ arbitrary if $k>j$.

\footnotetext{
${ }^{1}$ For existence of the optimal it is common [10] to assume that $Q=Q^{\prime} \geqslant 0, F=F^{\prime} \geqslant 0$, and $\left[\begin{array}{cc}Q & S \\ S^{\prime} & I\end{array}\right] \geqslant 0$ on $\left[t_{0}, t_{1}\right]$, and $(A(t), B(t))$ controllable.
} 
Proof: It is sufficient to note that in the matrix $G^{-1}=\left(\gamma_{v i}\right)$ defined in $\left[9\right.$, proof of Lemma 8] the nonzero elements of $\gamma_{v i}$ appear for $1 \leqslant v \leqslant r_{p_{i}}$ only and we now have $p_{i} \equiv 1$ for each $i, r_{1}+1 \leqslant i \leqslant m$.

We now consider the controllable system where $A(t)$ and $B(t)$ are of the Brunovsky form, respectively, [9] with cost function having $S(t) \equiv 0$.

Because of the canonical form for $A(t)$ and $B(t)$ there are model equations of the form $\dot{x}_{i}=x_{i+1}$ corresponding to l's on the super diagonal of $A(t)$ and 0 's in $B(t)$. In this case consider the term $q_{i, i+1}(t) x_{i} x_{i+1}$ of the cost function. So

$$
\begin{aligned}
\int_{t_{0}}^{t_{1}} 2 q_{i, i+1}(t) x_{i} x_{i+1} d t & =\int_{t_{0}}^{t_{1}} 2 q_{i, i+1}(t) x_{j} \dot{x}_{i} d t \\
& =\left.q_{i, i+1}(t) x_{i}^{2}\right|_{t_{0}} ^{t_{1}}-\int_{t_{0}}^{t_{1}} \dot{q}_{i, i+1}(t) x_{i}^{2} d t
\end{aligned}
$$

which contains terms that depend only on the endpoints at $t_{0}$ and $t_{1}$ and a term which can be added to the $q_{i i}(t) x_{i}^{2}$ term in $x^{\prime} Q(t) x$.

$$
i=\sum_{k=1}^{s} p_{k}, \quad j=\sum_{k=1}^{t} p_{k} \quad \text { for } s \neq t, \quad 1 \leqslant s, t \leqslant m .
$$

For example,

$$
\begin{aligned}
\int_{t_{0}}^{t_{1}} 2 q_{p_{1}, p_{1}+p_{2}}(t) x_{p_{1}} & x_{p_{1}+p_{2}} d t \\
= & \int_{t_{0}}^{t_{1}}\left\{\xi_{1}(t) x_{1} x_{p_{1}+1}+\xi_{2}(t) x_{1} x_{p_{1}+p_{2}+1}+\cdots\right. \\
& \left.+\xi_{m-1} x_{1} x_{p_{1}+\cdots+p_{m-1}+1}\right\} d t+S_{1}+S_{2}
\end{aligned}
$$

where $\xi_{j}(t)(1 \leqslant j \leqslant m-1)$ are some known functions, $S_{1}$ are the terms which can be added to $2 x^{\prime} S(t) u$ and $S_{2}$ terms which involve the values of $x(t)$ at $t=t_{0}$ and $t=t_{1}$ only.

Eventually, the symmetric matrix $Q(t)$ could be reduced to the form of

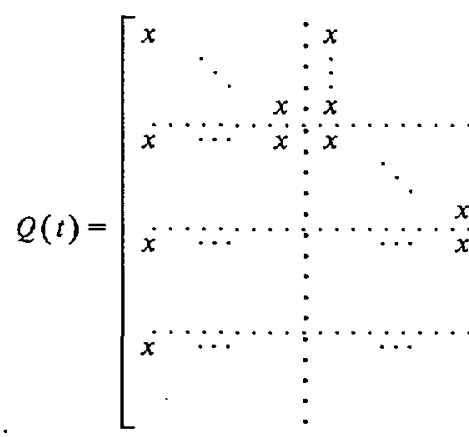

Similarly, we can treat terms of the cost function of the form $q_{i, i+l}(t) x_{i} x_{i+l}, l=2,3, \cdots$, if $x_{i+l}=\dot{x}_{i+l-1}$ for then

$$
\begin{aligned}
\int_{t_{0}}^{t_{1}} q_{i, i+l}(t) x_{i} x_{i+l} d t \\
\quad=\int_{t_{0}}^{t_{1}} q_{i, i+l} x_{i} \dot{x}_{i+l-1} d t \\
\quad=\left.q_{i, i+l} x_{i} x_{i+l-1}\right|_{t_{0}} ^{t_{1}}-\int_{t_{0}}^{t_{1}} x_{i+l-1}\left[\dot{q}_{i, i+l}(t) x_{i}+q_{i, i+l} \dot{x}_{i}\right] d t
\end{aligned}
$$

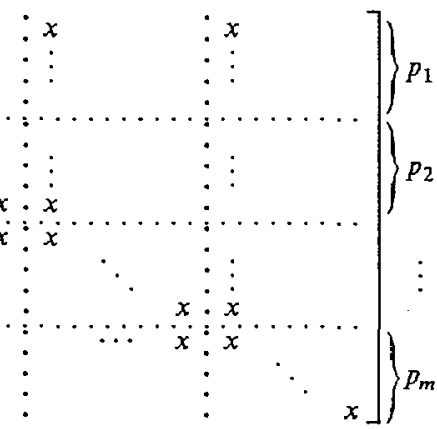

so that the number of independent elements in $Q(t)$ is at most $\sum_{j=1}^{m}(m-$ $j+1) p_{j}$. The total number of parameters (functions) in $A, B, Q, S$, and $R$ is at most $2 m n+(m(m-1) / 2)+\sum_{j=1}^{m}(m-j+1) p_{j}$.

More undefined elements in $Q(t), B(t)$, and $A(t)$ could be cancelled whenever $r_{1}<r_{0}$. As a matter of fact, Lemma 2.1 indicates that the number of the independent elements in $B(t)$ then is at most $r_{1}\left(2 m-r_{1}-\right.$ 1) $/ 2$ rather than $m(m-1) / 2$. The matrix $Q(t)$ in this case will have the form of

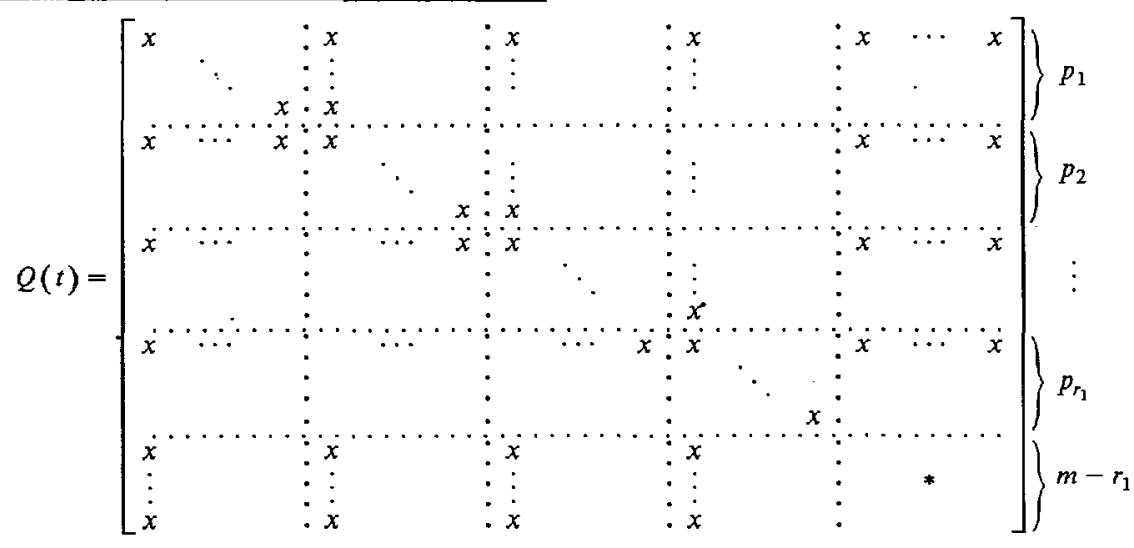

For $l=2$ this leads to terms which were met before if there is the relationship $\dot{x}_{i}=x_{i+1}$. The case of $l=3$, and so on are similar. In this fashion we can eliminate a total of $(n-m)(n-m+1) / 2$ functions from $Q(t)$.

The total number of functions in $A, B, Q$, and $R$, is

$$
m n+\frac{m(m-1)}{2}+\left[\frac{n(n+1)}{2}-\frac{(n-m)(n-m+1)}{2}\right]=2 m n .
$$

In addition there are $n(n+1) / 2$ constants in $x^{\prime}\left(t_{1}\right) F x\left(t_{1}\right)$.

It is noted that in case $S(t) \neq 0$ even more elements in $x^{\prime} Q x$ might be eliminated. In fact, using the same technique, one can deal with terms of the form $q_{i j}(t) x_{i} x_{j}$ for which which has at most

$$
\sum_{j=1}^{r_{1}}\left(r_{1}-j+1\right) p_{j}+\frac{\left(m-r_{1}\right)\left(m+r_{1}+1\right)}{2}
$$

elements other than zeros.

We now are in a position to deal with the system matrix $A$ further. We consider the time-invariant case, a slight modification for time-variant case will be given later.

A reordering of the state variable, by an orthogonal transformation $\bar{x}=J x$ yields 


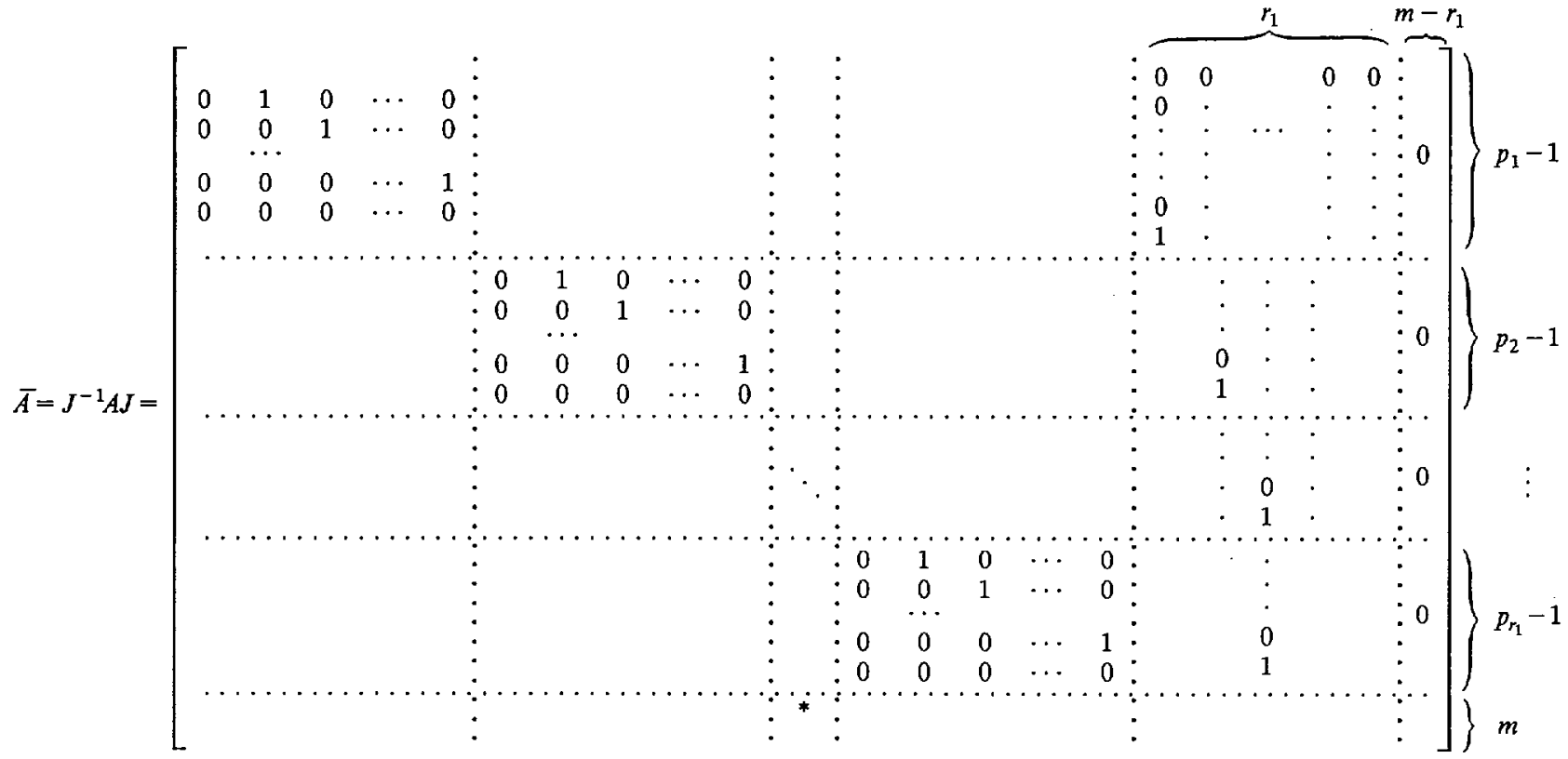

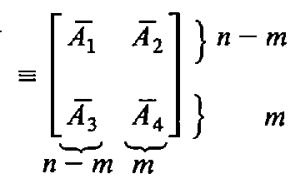
and

$$
\begin{aligned}
\bar{B} & =J^{-1} B=\left[\begin{array}{ccccccccc}
\ldots & \ldots & \ldots & \ldots & 0 & \ldots & \ldots & \ldots & \ldots \\
1 & x & x & x & \cdots & x & x & x & x \\
0 & 1 & x & x & \cdots & x & x & x & x \\
0 & 0 & 0 & 0 & \cdots & 1 & x & x & x \\
0 & 0 & 0 & 0 & \cdots & 0 & 1 & \cdots & 0 \\
0 & 0 & 0 & 0 & \cdots & 0 & 0 & \cdots & 1
\end{array}\right] \\
& \left.=\left[\begin{array}{c}
0 \\
\bar{B}_{1}
\end{array}\right]\right\} \begin{array}{ll}
m \\
n-m
\end{array}
\end{aligned}
$$

Now seek a nonsingular transformation $T$ of the coordinates of state $x$ that will maintain the forms of $\bar{A}$ and $\bar{B}$ and zero as many as possible elements in $\overline{A_{3}}$ and $\overline{A_{4}}$.

To preserve the form of $\bar{B}, T$ should be a block-lower triangle matrix. Indeed, it could be seen that taking $T=\left[\begin{array}{cc}I_{n-m} & 0 \\ T_{2} & I_{m}\end{array}\right]$ will yield

$$
\begin{aligned}
& \hat{A}=T \bar{A} T^{-1}=T \bar{A} P P^{-1} T^{-1}=\left[\begin{array}{cc}
I_{n-m} & 0 \\
T_{2}+A_{3}^{*} & A_{4}^{*}
\end{array}\right] P^{-1}\left[\begin{array}{cc}
I_{n-m} & 0 \\
-T_{2} & I_{m}
\end{array}\right] \\
& =\left[\begin{array}{ll}
\overline{A_{1}} & \overline{A_{2}} \\
H_{2} & H_{2}
\end{array}\right]\left[\begin{array}{cc}
I_{n-m} & 0 \\
-T_{2} & I_{m}
\end{array}\right]=\left[\begin{array}{cc}
\overline{A_{1}}-\overline{A_{2}} T_{2} & \overline{A_{2}} \\
H_{1}-H_{2} T_{2} & H_{2}
\end{array}\right] \text { and } \hat{B}=T \bar{B}=\left[\begin{array}{c}
0 \\
\bar{B}_{1}
\end{array}\right]
\end{aligned}
$$

where $P$ as an elementary matrix changes columns of $\bar{A}$ so that $\left[\begin{array}{ll}\overline{A_{1}} & \overline{A_{2}}\end{array}\right]$ will become $\left[\begin{array}{ll}I_{n-m} & 0\end{array}\right],\left[\begin{array}{ll}A_{3}^{*} & A_{4}^{*}\end{array}\right]=\left[\begin{array}{ll}\bar{A}_{3} & \bar{A}_{4}\end{array}\right] P$ and $\left(\left[\begin{array}{ll}H_{1} & H_{2}\end{array}\right]\right)=\left[T_{2}+\right.$ $\left.\left.A_{3}^{*} A_{4}^{*}\right]\right) P^{-1}$. So we require that $\bar{A}_{2} T_{2}=0_{n-m}$. Note that $\bar{A}_{2}=\left[\begin{array}{ll}\bar{A}_{21} & 0\end{array}\right]$ with $\bar{A}_{21}$ full rank. Thus, with commensurate partitioning, one has

$$
0_{n-m}=\left[\begin{array}{ll}
A_{21} & 0
\end{array}\right]\left[\begin{array}{l}
T_{21} \\
\bar{T}_{2}
\end{array}\right]=A_{21} T_{21}
$$

which implies $T_{21}=0$, i.e., $T_{2}=\left[\begin{array}{c}0 \\ \bar{T}_{2}\end{array}\right]$ where the elements of $\bar{T}_{2} \in$
$R^{\left(m-r_{1}\right) \times(n-m)}$ can be chosen freely. We now have

$$
\hat{A}=\left[\begin{array}{cc}
\overline{A_{1}} & \overline{A_{2}} \\
H_{1}-H_{2} T_{2} & H_{2}
\end{array}\right]=\left[\begin{array}{ll}
\overline{A_{1}} & \overline{A_{2}} \\
\hat{A_{3}} & \hat{A}_{4}
\end{array}\right] .
$$

Note that matrix $H_{2}$ consists of the last $\left(m-r_{1}\right)$ columns of $A_{4}^{*}$, which we will denote by $A_{4}^{* *}$ and $r_{1}$ columns of $T_{2}+A_{2}^{*}$ which will be denoted by $T_{2}^{*}+A_{2}^{* *}$ where $T_{2}^{*}$ consists of some $r_{1}$ columns of $T_{2}$ (exactly they are the $\left(k_{i}-1\right)$ th columns $\left(1 \leqslant i \leqslant r_{1}\right)$ of $\left.T_{2}\right)$ and $A_{2}^{* *}$ consists of corresponding $r_{1}$ columns of $A_{2}^{*}$, i.e.,

$$
H_{2}=[\overbrace{T_{2}^{*}+A_{2}^{* *}}^{r_{1}} \overbrace{A_{4}^{* *}}^{m-r_{1}}] .
$$

Thus,

$$
T_{2}^{*}+A_{2}^{* *}=\left[\begin{array}{c}
A_{21}^{* *} \\
T_{2}^{* *}+A_{22}^{* *}
\end{array}\right],
$$

therefore taking $T_{2}^{* *}=-A_{2}^{* *}$ will produce $r_{1}\left(m-r_{1}\right)$ zero elements in $\mathrm{H}_{2}$ and in $\hat{A}$.

Furthermore, in case $n>m+r_{1}$, more zero elements could be obtained by choosing other free elements in $T_{2}$. To do this; we observe that $\hat{A_{3}}=H_{1}-H_{2} T_{2}=H_{1}-A_{4}^{* *} \bar{T}_{2}$ where, because of $T_{2}^{* *}=-A_{2}^{* *}$ the $\left(p_{i}\right.$ -1)th columns $\left(1 \leqslant 1 \leqslant r_{1}\right)$ of $A_{4}^{* *} \bar{T}_{2}$ have been fixed, and by $\left[H_{1}\right.$ $\left.H_{2}\right]=\left[T_{2}+A_{3}^{*}: A_{4}^{*}\right] P^{-1}$ (because of the existence of $P^{-1}$ ), however, each $\left(p_{i}-1\right)$ th column $\left(1 \leqslant i \leqslant r_{1}\right)$ of $H_{1}$ now contains $\left(p_{i}-2\right)$ th column $\left(1 \leqslant i \leqslant r_{1}\right)$ of $\bar{T}_{2}$ whose elements still could be chosen freely. Hence, if $n \geqslant m+2 r_{1}$, then choosing free elements in $T_{2}$ appropriately will give $r_{1}\left(m+r_{1}\right)$ zero elements in $\hat{A}_{3}$. By a similar analysis, if $m+r_{1}<n<m+$ $2 r_{1}$, then choosing some free elements in $T_{2}$ will get $\left[n-\left(m+r_{1}\right)\right]\left(m-r_{1}\right)$ zero elements in $\hat{A}_{3}$.

In the time-variant case, transformation matrix $T(t)$ could still be chosen as

$$
T(t)=\left[\begin{array}{cc}
I_{n-m} & 0 \\
T_{2}(t) & I_{m}
\end{array}\right] \quad \text { where } \quad T_{2}(t)=\left[\begin{array}{c}
0 \\
\bar{T}_{2}
\end{array}\right]
$$

Thus,

$$
\hat{A}(t)=T \bar{A} T^{-1}+\dot{T} T^{-1}=\left[\begin{array}{cc}
\overline{A_{1}}(t) & \overline{A_{2}}(t) \\
H_{1}-H_{2} T_{2}+\dot{T}_{2} & H_{2}
\end{array}\right]
$$

where $\left[\begin{array}{ll}H_{1} & H_{2}\end{array}\right] \equiv\left[\begin{array}{ll}T_{2}+A_{3}^{*} & A_{4}^{*}\end{array}\right] P^{-1}, P$ is defined above and $\left[A_{3}^{*}\right.$ $\left.A_{4}^{*}\right]=\left[\begin{array}{ll}\overline{A_{3}} & \bar{A}_{4}\end{array}\right] P$. Therefore, the same arguments as before could also be carried out here. 
TABLE I

\begin{tabular}{|c|c|c|c|c|}
\hline \multirow{3}{*}{$\begin{array}{l}\text { Dimensions } \\
\text { Matrices }\end{array}$} & \multirow{3}{*}{$m=n$} & \multicolumn{3}{|c|}{$m<n$} \\
\hline & & \multirow{2}{*}{$r_{1}=m$} & \multicolumn{2}{|r|}{$r_{1}<m$} \\
\hline & & & $n<m+2 r_{1}$ & $n>m+2 r_{1}$ \\
\hline$A(t)$ & $n^{2}$ & $\mathrm{~m} n$ & $m n-(n-m)\left(m-r_{1}\right)$ & $m n-2 r_{1}\left(m-r_{1}\right)$ \\
\hline$B(t)$ & 0 & $\frac{\underline{m}(m-1)}{2}$ & $\frac{m(m-1)}{2}-\frac{\left(m-r_{1}-1\right)\left(m-r_{1}\right)}{2}$ & $\frac{m(m-1)}{2}-\frac{\left(m-r_{1}-1\right)\left(m-r_{1}\right)}{2}$ \\
\hline$Q(t)$ & $\frac{n(n+1)}{2}$ & $\sum_{j=1}^{m}(m-j+1) p_{j}$ & $\sum_{j=1}^{r_{1}^{1}}\left(r_{1}-j+1\right) p_{j}+\frac{\left(m-r_{1}\right)\left(m+r_{1}+1\right)}{2}$ & $\sum_{j=1}^{r_{1}}\left(r_{1}-j+1\right) p_{j}+\frac{\left(m-r_{1}\right)\left(m+r_{1}+1\right)}{2}$ \\
\hline$s(t)$ & $n^{2}$ & $\mathrm{mn}$ & an & $\mathrm{mn}$ \\
\hline $\mathrm{F}$ & $\frac{n(n+1)}{2}$ & $\frac{n(n+1)}{2}$ & $\frac{n(n+1)}{2}$ & $\frac{n(n+1)}{2}$ \\
\hline
\end{tabular}

Transformation $T(t)$, defined above, preserves the forms of $\hat{A}$ and $\hat{B}$ to be like those of $\bar{A}$ and $\vec{B}$, respectively, hence the technique used for reducing $Q(t)$ is still available in the present case. It should be observed that for $T(t)$ defined above we have det $T(t) \equiv 1$ and its elements involve some elements of $A(t)$ and their first derivatives only which implies that $T(t)$ is a Lyapunov transformation provided $A(t) \in C^{2}$ on $\left[t_{0}, t_{1}\right]$.

We now summarize these results as follows.

Theorem 2.I: Assume that the system is controllable and $B(t)$ is of full rank on $\left[t_{0}, t_{1}\right]$. The functions $r_{0}(t), \cdots, r_{n-1}(t)$ defined above are assumed to be constants on $\left[t_{0}, t_{1}\right]$ and $r_{0}(t)+\cdots+r_{n-1}(t)=n$. The upper bounds of the independent parameters in the integral quadratic cost formulation can then be described as in Table I.

\section{Independent Parameters in $F$-EQuivalent Class}

In this section we restrict attention to the case $r_{1}=r_{0}(=m)$ (the extension to the case $r_{1}<r_{0}$ is straightforward but somewhat more involved.)

For the $F$-equivalent class, we can take the system $\dot{x}=\bar{A} x+\bar{B} u$ with the cost functional as our starting point, where $\bar{A}$ and $\bar{B}$ were described above with $r_{1}=m$, respectively. We now apply a linear state feedback controller $u=G x+v$ in the system, which leads to the closed-loop system $\dot{x}=(\bar{A}+\bar{B} G) x+\bar{B} v$ with the cost functional

$$
\int_{t_{0}}^{t_{1}}\left(x^{\prime} \bar{Q} x+2 x^{\prime} \bar{S} v+v^{\prime} v\right) d t+x^{\prime}\left(t_{1}\right) F x\left(t_{1}\right)
$$

where

$$
\bar{Q}=Q+S G+G^{\prime} S^{\prime}+G^{\prime} G, \bar{S}=S+G^{\prime}
$$

and

$$
\bar{A}+\bar{B} G=\left[\begin{array}{c}
\overline{A_{1}}: \overline{A_{2}} \\
\cdots \\
Y
\end{array}\right]
$$

where $Y=\left[\begin{array}{ll}\overline{A_{3}} & \overline{A_{4}}\end{array}\right]+\bar{B}_{1} G$.

It should be noted that $\bar{B}_{1}$ is invertible and det $\bar{B}_{1} \equiv 1$ which implies that for any specified $m \times n$ matrix $Y^{*}(t)$ one can always find an appropriate feedback gain matrix $G=\bar{B}_{1}^{-1}\left(Y^{*}(t)-\left[\begin{array}{ll}\overline{A_{3}} & \overline{A_{4}}\end{array}\right]\right)$ so that the resulting closed-loop system has the matrix

$$
\bar{A}=\left[\begin{array}{cc}
\overline{A_{1}} & \overline{A_{2}} \\
\hdashline Y^{*}
\end{array}\right] \equiv\left[\begin{array}{ll}
\overline{A_{1}} & \overline{A_{2}} \\
Y_{1}^{*} & Y_{2}^{*}
\end{array}\right]
$$

as its system matrix. It can also be seen that a suitable choice of $G$ would make $\overline{\bar{A}}$ be a stable constant matrix even though $\bar{A}$ is a time-variant one.

Having done this, we now replace $P(t)$ in the corresponding Riccati differential equation by $\bar{P}(t)=P(t)+L(t)$ which leads to cost functional

$$
\int_{t_{0}}^{t_{1}}\left(x^{\prime} \overline{\bar{Q}} x+2 x^{\prime} \overline{\bar{S}} v+v^{\prime} v\right) d t+x^{\prime}\left(t_{1}\right) \overline{\bar{F}} x\left(t_{1}\right)
$$

where $\overline{\bar{Q}}=\bar{Q}-\overline{\overline{A^{\prime}}} L-L \overline{\bar{A}}-\dot{L}, \overline{\bar{S}}=\bar{S}-L \bar{B}$, and $\overline{\bar{F}}=F+L\left(t_{1}\right)$.

It can be easily checked that matrix $L(t)$ specified by

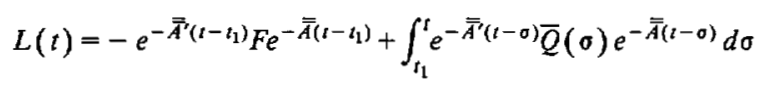

makes $\overline{\bar{Q}}=0$ and $\overline{\bar{F}}=0$ so that the cost function becomes $2 \int_{t_{0}}^{t_{1}}\left[x^{\prime} \overline{\bar{S}} v+\right.$ $\left.v^{\prime} v\right] d t$. Thus, the parameters appear in $\overline{\bar{S}}$ and $\bar{B}$ only and have total number $\eta_{1}=m n+(m(m-1) / 2)$. (In the single input case $n$ independent parameters.)

It should be noted that by using transformations ${ }^{2} C, G, D$ and $L$, the number of the invariants for the quadratic cost problem which can be characterized by $(A, B, Q, S, R, F)$ should be equal to

$$
\begin{aligned}
\eta_{2}= & {\left[n^{2}+m n+\frac{n(n+1)}{2}+m n+\frac{m(m+1)}{2}\right] } \\
& -\left[n^{2}+m n+m^{2}+\frac{n(n+1)}{2}\right] \\
= & m n-\frac{m(m-1)}{2}
\end{aligned}
$$

which is strictly less than $\eta_{1}$ defined above except for $m=1$.

Theorem 3.1: For a controllable time-variant system with $r_{1}=r_{0}=m$, its quadratic cost formulation can be reduced to another formulation in the $F$-equivalent class which has at most $m n+(m(m-1) / 2)$ independent parameters (functions).

\footnotetext{
${ }^{2}$ The set of transformations $\{C, G, D, L\}$ is labeled the optinal feedback group.
} 


\section{REFERENCES}

11] R. E Kalman, "When is a linear control system optimal?" Trans. ASME J. Bosic Eng., vol. 86, pp. 51-60, 1964

[2] W. M. Wonham and C. D. Johnson, "Optimal bang-bang control with quadratic performance index," Trans. ASME J. Basic Eng., vol. 86, pp. 107-115, 1964.

[3] H. R. Sirisena, "Optimal control of saturating linear plants for quadratic performance indices," Int. J. Contr., vol. 8, pp. 65-87, 1968.

[4] E. Kreindler and J. K. Hedrick, "On equivalence of quadratic loss functions," Int. J. Contr., vol. 11, no. 2, pp. 213-222, 1970.

[5] E. B. Lee, "ONR task 3 results: There are at most $2 \mathrm{~nm}$ real parameters in the quadratic cost problem," Honeywell Inc., Tech. Rep. MR12353, June 1975.

[6] C. A. Harvey, "Independent parameters in quadratic performance indices," Honeywell Inc., Tech. Rep. MR12366, June 1975.

[7] E. B. Lee, "Independent functions in the time varying quadratic cost problem," Honeywell Inc., Tech. Rep. MR 12368, July 1975.

[8] E. B. Lee and W. S. Lu, "On independent parameters in quadratic cost problem, Part I: Constant case; Part II: Time-variant case," Univ. Minnesota, Res. Rep., 1981.

[9] P. Brunovsky, "A classification of linear controllable systems," Kybernetika Cislo, vol. 3, pp. 173-188, 1970.

[10] R. W. Brockett, Finite Dimensional Linear System. New York: Wiley, 1970.

\section{A Note on Trajectory Sensitivity Reduction Using a Three-Term Controller}

\section{H. KHALIFA AND A. A. R. HANAFY}

\begin{abstract}
This technical note investigates the effectiveness of using a three-term controller for a linear quadratic regulator (LQR) problem in reducing trajectory sensitivity to plant parameters variation as compared to other developed techniques to solve the same problem.
\end{abstract}

\section{INTRODUCTION}

The optimization of the linear quadratic regulator (LQR) problem, together with trajectory sensitivity minimization, has been investigated by several authors [1]-[4].

The conventional approach has been to construct a proportional controller ( $P$-controller) where feedback is taken from both the plant states as well as the sensitivity functions generated from an augmented sensitivity model. Closed-loop sensitivity functions are to be used in order to effectively reduce the trajectory sensitivity to plant parameters variation. However, the complexity of the computation algorithm, together with the increase in the dimension of the optimization problem, makes this approach impractical.

In [4], the original LQR problem is solved where the states' quadratic term in the performance index is multiplied by a scalar $\alpha$. As $\alpha$ is increased from unity, a tradeoff between the increase in the performance index and the reduction in the value of a sensitivity index results in a suitable value for $\alpha$, and the suboptimal state feedback $P$-controller has been obtained. However, this algorithm is based on the proposition that the resulting reduction in the sensitivity index is much greater than the resulting increase in the performance index value $-a$ proposition that will be shown to not always be true.

In this note, the effectiveness of a three-term (PID) controller in reducing trajectory sensitivity is investigated.

\section{DESIGN OF A PID CONTROLLER}

Consider the plant model

$$
\begin{aligned}
& \dot{x}(t)=A x(t)+B u(t) \quad x(0)=x_{0} \\
& y(t)=C x(t)
\end{aligned}
$$

Manuscript received January 17, 1983; revised June 1, 1983, July 12, 1983, and December 13,1983

The authors are with the Department of Electrical Engineering, Cairo University, Gizah, Esypt. where $x(t), u(t)$, and $y(t)$ are $n$-state vector, $m$-control vector, and l-output vector, respectively.

Using the augmented model

$$
\dot{z}(t)=A z(t)+B u(t), \quad z(0)=\left[\frac{x(0)}{0}\right]
$$

where

$$
z(t)=\left[\frac{x(t)}{v(t)}\right], \quad A=\left[\begin{array}{l|l}
A & 0 \\
\hline D & 0
\end{array}\right], \quad B=\left[\frac{B}{0}\right] .
$$

The matrix $D$ is an $r \times n$-matrix, where $r=m$. A necessary and sufficient condition for the augmented system described by (2) to be completely controllable is that the pair $(A, B)$ be controllable and the rank of $D A^{-1} B=r$. If $A$ is singular, then the augmented system is still controllable if $(A, B)$ is controllable and a stabilizing matrix $F$ can be found such that $(A+B F)$ is nonsingular and the rank of $D(A+B F)^{-1} B$ $=r$. These conditions have already been stated in [5].

Then, the optimum control $u^{*}(t)$ that would minimize the index

$$
\hat{J}=\frac{1}{2} \int_{0}^{\infty}\left(z^{T}(t) \hat{Q}_{z}(t)+u^{T}(t) R u(t)\right) d t
$$

where $\hat{Q}=\operatorname{diag}\left[Q, Q_{I}\right]$, is a positive semidefinite diagonal matrix and $R$ is a positive definite matrix, is given by

$$
u^{*}(t)=-K^{*} z(t)=-R^{-1} B^{T} P z(t)
$$

where

$$
0=\hat{P} \hat{A}+\hat{A}^{T} \hat{P}-\hat{P} \hat{B} R^{-1} \hat{B}^{T} \hat{P}+\hat{Q}
$$

and

$$
K^{*}=\left[K_{p}^{*}: K_{I}^{*}\right]
$$

Assuming $u^{*}(t)$ to have the alternative structure

$$
u^{*}(t)=-K_{p} y_{1}(t)-K_{I} \int_{0}^{t} y_{2}(t) d t-K_{D} \dot{y}_{3}(t)
$$

where

$$
y_{i}(t)=L_{i} y(t), \quad i=1,2,3
$$

and $L_{i}$ is a matrix of dimension $\left(l_{i} \times 1\right)$ satisfying

$$
l_{i} \leqslant l, \quad l_{1}+l_{3} \leqslant n, \quad \sum_{i} l_{i} \leqslant n+m .
$$

The selection of the matrix $D$ is constrained only by the controllability condition for the augmented system, and that of the matrices $L_{i}$ is decided by the designer experience.

Substituting for $u(t)$ in (1) using (4) and (6), the following relations result:

$$
\begin{aligned}
{\left[K_{p}: K_{D}\right] } & =K_{p}^{*} \Gamma^{T}\left(\Gamma \Gamma^{T}\right)^{-1} \\
K_{I} & =\left(I+K_{D} L_{3} C B\right) C^{T} L_{2}^{T}\left(L_{2} C C^{T} L_{2}^{T}\right)^{-1}
\end{aligned}
$$

where

$$
\Gamma \triangleq\left[\frac{L_{1} C}{L_{3} C A-L_{3} C B K_{p}^{*}}\right]
$$

\section{EXAMPLE}

The following system is considered: 